Journal of Computer Science 8 (1): 13-17, 2012

ISSN 1549-3636

(C) 2011 Science Publications

\title{
Mobility and Density Aware AODV Protocol Extension for Mobile Adhoc Networks-MADA-AODV
}

\author{
${ }^{1}$ S. Deepa and ${ }^{2}$ G.M. Kadhar Nawaz \\ ${ }^{1}$ Department of MCA, Bharathiyar University,Coimbatore, India \\ ${ }^{2}$ Sona Technology, Salem, India
}

\begin{abstract}
Problem statement: In this study, we proposed a method to improve the performance of AODV by reducing overhead by adopting a route message propagation mechanism. According to the new scheme, a node will be selected to forward a routing request message if and only if a condition based on its mobility (speed) and number of neighbors is satisfied. If the routing request is allowed to propagate through a node then there will be at least a possible path which includes that node in its path list. So, at the end of the route resolving process, the destination will have a possible path through that node. If the routing request is disallowed to propagate through a node then there will not be a possible path which includes that node in its path list. So, at the end of the route resolving process, the destination will not have a possible path through that node. We implemented the idea on network simulator (ns2) and measured the improvement in performance. Mobility and node density are the two major factors which has much influence on the performance of any routing protocol of mobile adhoc network. Several previous works highlighted this fact. In this study, we will improve the performance of AODV by adding mobility and density aware behaviors in route resolving process. Approach: In this study we describe an idea for improving the performance of AODV and reduce some of the overheads in a large and dense network with mobile nodes with different speeds. The proposed design will be implemented on AODV as an extension and will give a new protocol namely 'Mobility and Density Based Extended AODV' (MADA-AODV). Results: The performance of MADA-AODV has been compared with the other routing protocols AODV,DSDV and DSR with metrics throughput, MAC load, Routing Load, Control message overhead etc.,. will be used to measure the performance of the protocol in terms of different network overhead. We have arrived more significant and comparable results. Conclusion: We have successfully implemented the proposed protocol MADA-AODV and compared it with other routing protocols AODV, DSDV, DSR. We evaluated the performance of these protocols in terms of different metrics. In almost all the cases, the performance of MADA-AODV is better than normal AODV as well as other compared routing protocols. Significant improvement in throughput as well as significant reduction in overhead is provided by MADA-AODV. So we hereby conclude that MADAAODV will be suitable for highly mobile and dense network scenario.
\end{abstract}

Key words: Mobile adhoc network, routing overhead, route discovery, mobility aware routing, routing protocols, DSDV, DSR, AODV, MADA-AODV

\section{INTRODUCTION}

A Mobile Ad-Hoc Network (MANET) is a selfconfiguring network and the nodes are connected through wireless link. It is an infrastructure less network. The wireless network topology may change rapidly. Each node in the network act as router and it communicate other nodes. There is no centralized administration. Nodes in ad hoc networks are differentiated by their limited resources like power, memory and mobility. Due to the limited transmission range of the nodes, multiple hops may be needed for a node to send data to any other node in the network. Thus each node acts as a host and router. If a node needs to communicate with another that is outside its transmission range, an intermediate node acts as a router to relay or forward packets from the source to the destination. For this purpose, a routing protocol is needed. Routing protocol design is an important and essential issue for Ad Hoc networks due to dynamism of the network. One interesting research area in MANET is routing. Routing in the MANETs is a challenging task and has received a tremendous amount of attention from researches.

\section{MATERIALS AND METHODS}

Guaranteeing delivery and the capability to handle dynamic connectivity are the most important issues for 
routing protocols in wireless mobile ad hoc networks. Once there is a path from the source to the destination for a certain period of time, the routing protocol should be able to deliver data via that path. If the connectivity of any two nodes changes and routes are affected by this change, the routing protocol should be able to recover if an alternate path exists.

Different types of communications used in mobile ad hoc networks are:

- Unicasting

- Broadcasting

- Multicasting

- Anycasting

Unicasting: Unicast transmission is between one-toone nodes only two nodes are exchanging the informations.

Broadcasting: Broadcast is a type of transmission in which information is sent from just one node but is received by all the nodes connected to the network. One to all communication is called as broadcast.

Anycasting: Anycast is communication between a single sender and several receivers topologically nearest in a group. The term exists in contradistinction to multicast, communication between a single sender and a group of selected receivers.

Multicasting: Multicast is a very much different from Unicast. It is a type of transmission or communication in which there may be more than one nodes and the information sent to a set of nodes. It is a limited case of broadcasting. Multicasting is used within the network has many advantages. Multicasting reduces communication cost for applications that send the same data to more recipients.

Types of MANET routing protocols: Routing protocols are classified into two types based on their Properties.

- $\quad$ Proactive routing protocols

- Reactive routing protocols

Table driven routing protocols (Proactive): In proactive or table-driven routing protocols, each node continuously maintains up-to-date routes to every other node in the network. Routing information is periodically transmitted throughout the network in order to maintain routing table consistency. The areas in which they differ are the number of necessary routing-related tables and the methods by which changes in network structure are broadcast. The proactive protocols are not suitable for larger networks, as they need to maintain node entries for each and every node in the routing table of every node.

On-demand routing protocols (Reactive): With ondemand protocols, if a source node requires a route to the destination for which it does not have route information, it initiates a route discovery process which goes from one node to the other until it reaches to the destination or an intermediate node has a route to the destination. If a node wants to send a packet to another node then this protocol searches for the route in an ondemand manner and establishes the connection in order to transmit and receive the packet. The route discovery usually occurs by flooding the route request packets throughout the network.

This study examines routing protocols designed for these ad hoc networks by first describing the classification of ad hoc routing protocols

About study: Mobility and node density are the two major factors which has much influence on the performance of any routing protocol of mobile ad hoc network. All the overheads such as MAC layer overheads and Network Layer will get worse very much while increasing the mobility of the nodes and the node density of the network. In study, we will measure the performance of four MANET routing protocols with different mobility and node density.

About the other compared routing protocols: Dynamic Source Routing (DSR): Dynamic source routing is a on demand routing protocol for Mobile Ad hoc Network and is based on the concept of source routing. The protocol smaintains route cache in each node which is updated when new routes are learned. The protocol consists of two phases. Route discovery and route maintenance. The source node broadcasts a Route Request (RR) packet consist of the destination node address, source node address and unique request ID. Each node receives the packet checks whether if route is available or not. If does not, it adds its own address to the route record and forwards the packet. Route Maintenance is achieved through the use of Route Error Packet (REP) and acknowledgements. Route error packets are generated at a node due to the problem of fatal transmission at the data link layer. When a route error packet is received, the hop in error is removed from the node's route cache and all routes containing the truncated at the point (Table 1). 
Table 1: Comparison of three routing protocols (Perkins et al., 2001)

\begin{tabular}{llll}
\hline Protocol property & DSDV & AODV & DSR \\
\hline Routing type & Flat & Flat & Flat \\
Routing metric & Shortest path & Fresh and shortest path & Shortest path \\
Routing maintenance & Routing table & Routing table & Routing cache \\
Multiple route & No & No & Yes \\
Loop free & Yes & Yes & Yes \\
Multicast periodic & No & Yes & No \\
Broadcast & Yes & Yes & No \\
\hline
\end{tabular}

Destination-Sequenced Distance-Vector (DSDV): The destination sequenced distance vector routing protocol (Abd Rahman and Zukarnain 2009) is a proactive routing protocol based on the Bellman-Ford algorithm. Routing table is maintained at each node and with this table, node transmits the packet to other nodes in the network.

The different type of routing prtocols are compared based on the properties.

To guarantee loop-freedom DSDV uses a concept of sequence numbers to indicate the freshness of a route. The Broadcasting mechanism in the dsdv is of two types-Full dump and incremental dump. Full dump will carry all the routing information and the incremental dump will carry only last updation of full dump to improve the efficiency of the system. DSDV is not fit for large networks.

Ad hoc On-demand Distance Vector (AODV) routing: $A O D V$ is a reactive routing protocol (Perkins $e t$ al., 2001) implemented for mobile ad hoc networks. AODV is used for unicast, multicast and broadcast communication. AODV is combination of both DSR and DSDV. It adopts the basic on demand mechanism of Route Discovery and Route maintenance from DSR and the use of hop by hop routing sequence number and periodic beacons from DSDV. When a source node desires to sent information to destination node and does not have a route to destination, it starts the route discovery process.

It broadcasts RREQ to neighbors and then forward the request to their neighbors on so on up to route for the destination is located.And also send a route reply packet to the neighbors which is the first receives RREQ.RREP is routed along the reverse path. Each node maintains own sequence number and broadcast id. To maintain routes the nodes survey the link status of their next hop neighbor in active routes. If the destination or some intermediate node move, the node upstream of the break remove the routing entry and send Route Error (RERR) messages to affect the active route upstream neighbors. This continues until source node is reached.
The proposed MADA-AODV: The following sub sections explain the design and implementation of MAD-ADODV extension.

The basic design: A node will be selected to forward a routing request message if and only if a condition based on its mobility (speed) and number of neighbors is satisfied.

If the routing request is allowed to propagate through a node then there will be at least a possible path which includes that node in its path list. So, at the end of the route resolving process, the destination will have a possible path through that node.

If the routing request is disallowed to propagate through a node then there will not be a possible path which includes that node in its path list. So, at the end of the route resolving process, the destination will not have a possible path through that node.

The MADA-AODV extension: The node A starts a route resolve process for finding a path to the node $\mathrm{B}$ :

Any intermediate node Node $X$ receiving the routing request message will do the following:

- Calculate the present speed Sx of the node by using its present and previous location

- The node $\mathrm{X}$ will resolve the number of neighbors $\mathrm{Nx}$

- If $\mathrm{Nx}<\mathrm{Tn}$ then

Forward the packet unconditionally:

$$
\begin{aligned}
& \text { Else } \\
& \text { Calculate mobility factor Mp of } \mathrm{X} \\
& \mathrm{Mp}=\mathrm{f}(\mathrm{Sx})=\mathrm{Sx} * 1 / \mathrm{Se}-\text { will be } 0 \text { to } 1 \\
& \text { If } \mathrm{M}_{\mathrm{f}}<\mathrm{Mt} \text { then } \\
& \text { Forward the packet } \\
& \text { Else } \\
& \text { Do not Forward the packet } \\
& \text { End } \\
& \text { 'End }
\end{aligned}
$$

Where:

$\mathrm{N}_{\mathrm{x}}=$ Neighbors of node $\mathrm{X}$

$\mathrm{T}_{\mathrm{n}}=$ Minimum number of neighbors-if a node has neighbors less than this value then it will just forward the packer without future condition check

$\mathrm{M}_{\mathrm{f}}=$ Mobility factor

$\mathrm{M}_{\mathrm{t}}=$ Mobility threshold-A value which will decide the maximum speed of the node which will be allowed to forward the packet

$\mathrm{S}_{\mathrm{x}}=$ Speed of the node $\mathrm{X}$

$\mathrm{S}_{\mathrm{e}}=$ The maximum expected speed of a node 
Finally the node A will have a route through $\mathrm{X}$ if and only if that node $\mathrm{X}$ was allowed to forward the routing request message based on the previous condition.

So, as a net effect, the nodes which are moving very fast at that moment will be eliminated from the path. So the established paths of MADA-AODV will be stable than that of the paths resolved by normal AODV.

Simulation and metrics: The simulation tool that has been used in order to study all the behavior of MANET routing protocol was the ns2[http://www.isi.edu/nsnam/ns/, 1998, http://www.monarch.cs.cmu.edu/cmu-ns.html,

UCB/LBNL/VINT Network Simulator]The proposed simulation has been successfully done using ns 2 on a normal desktop computer with 1 GB RAM. CMU's wireless extension to ns-2 provides the implementation of the DSR, AODV, DSDV routing protocols.

\section{Parameters of the simulation: \\ Channel type \\ Antenna type \\ Interface queue type \\ MAC type \\ Max packet in queue \\ Topographical area \\ txPower \\ rxPower \\ idlePower \\ Routingprotocols \\ Node density \\ Mobility \\ With mobility scenario \\ Mobility model \\ Traffic parameters \\ Traffic \\ $\%$ of communicating nodes \\ CBR packet size \\ CBR interval \\ Maximum packets \\ Wireless channel \\ Two ray ground \\ Omni antenna \\ DropTail/PriQueue \\ 802_11 \\ 50 \\ $800 \times 800$ sq.m \\ $0.1819 \mathrm{~W}$ \\ $0.0501 \mathrm{~W}$
$0.0350 \mathrm{~W}$
DSDV/DSR/AODV/
MAD-AODV \\ $10,20,30,40,50 /$ \\ $800 \times 800$ sq m \\ $0-40 \mathrm{~m} \mathrm{sec}^{-1}$ \\ Random Waypoint \\ CBR over UDP \\ $50 \%$ \\ 512 Bytes \\ $0.1 \mathrm{sec}$ \\ 200}

We have tested the performance of the four routing protocols with different network size and with nodes of different speeds in all scenarios.

\section{Metrics considered for evaluation:}

Throughput: The throughput metric measures how well the network can constantly provide data to the sink. Throughput is the number of packet arriving at the sink per ms/second.
Mac load: The ratio of the number of MAC layer messages propagated by every node in the network and the number of data packets successfully delivered to all destination nodes. In other words, the MC load means the average number of MAC messages generated to each data packet successfully delivered to the destination.

Dropped packets: The Number of Nodes in the Network vs Agent level Total Dropped Packet is considered as the metric to analyze the performance.

\section{RESULTS}

The simulation results: The Fig. 1: graph shows throughput provided by the four different protocols with mobility and different node densities. As shown in the graph, the proposed MAD-AODV performed well in terms of throughput. Next to it, DSR performed good. DSDV is the poor performer in terms of throughput.

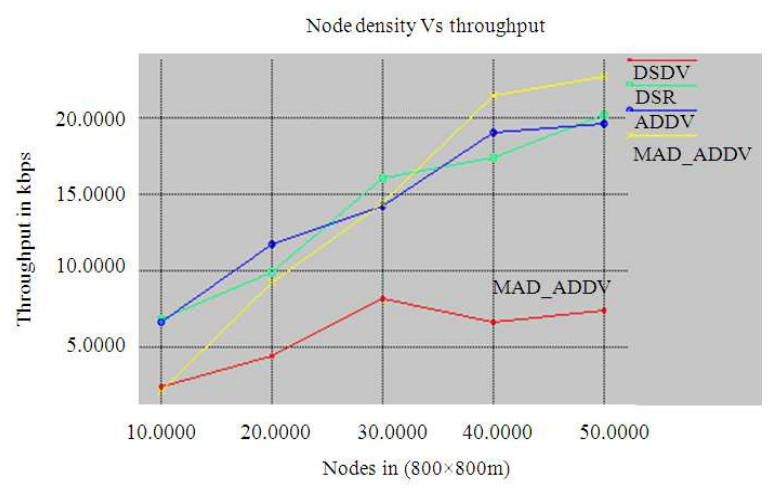

Fig. 1: The node density Vs throughput

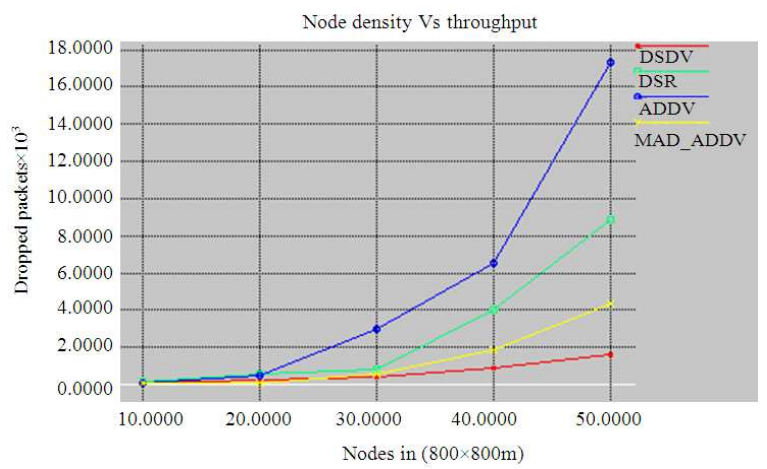

Fig. 2: The node density Vs dropped packets 


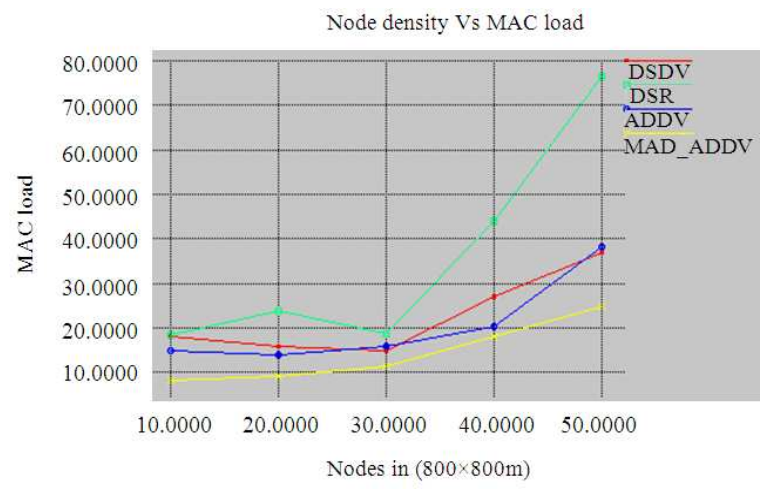

Fig. 3: The node density Vs MAC load

The Fig. 2: graph shows total dropped packet by the four different protocols with mobility and different node densities. As shown in the graph, the proposed MAD-AODV performed good and dropped less packets than the conventional AODV.

The Fig. 3 graph shows MAC load of the four different protocols with mobility and different node densities. As shown in the graph, MAD-AODV performed very good and caused less MAC load than the normal AODV.

\section{DISCUSSION}

If we carefully examine all the above graphs it is obvious that the proposed MAD-AODV is the protocol which is providing better performance than Normal AODV. In most cases, I have produced better results than all other compared protocols.

\section{CONCLUSION}

We have evaluated three commonly used adhoc routing protocols DSDV, DSR and AODV along with the proposed MADA-AODV protocol with different mobility and node density. If we carefully examine the graphs presented in previous section it is obvious that MADA-AODV is providing better performance than the normal AODV. And in most cases it outperformed all other compared protocols.

So future study may investigate the possibilities for further improving the routing mechanism of the proposed MADA-AODV. Presently, the core part of MADA-AODV algorithm will only consider the speed and neighbor density of the nodes during making decisions. Future study may address other possibilities like adding node velocity and relative location with respect to the sender and receiver as additional parameters during making decisions. There are endless possibilities to extend MADA-AODV to make it as a suitable candidate algorithm for mobile adhoc network. Future study may explore and address these possibilities.

\section{REFERENCES}

Perkins, C.E., E.M. Royer, S.R. Das, and M.K. Marina, 2001. Performance comparison of two on-demand routing protocols for Ad Hoc networks. IEEE Personal Commun. Magaz. Special Issue Ad hoc Networking, 8: 16-28.

Abd Rahman, A.H. and Z.A. Zukarnain, 2009. Performance comparison of AODV, DSDV and IDSDV Routing protocols in mobile ad hoc networks. Euro. J. Scientific Res., 31: 566-576. 